\title{
Use of Glycol Chitosan Modified by $5 \beta$-cholanic Acid Nanoparticles for the Sustained Release of Proteins during Murine Embryonic Limb Skeletogenesis
}

\author{
Tieshi Li ${ }^{1}$, Lara Longobardi ${ }^{1}$, Froilan Granero-Molto ${ }^{1}$, Timothy J. Myers ${ }^{1}$, Yun Yan ${ }^{1}$, and \\ Anna Spagnoli ${ }^{1,2, *}$ \\ ${ }^{1}$ Department of Pediatrics, University of North Carolina at Chapel Hill, Chapel Hill, NC 27599, \\ USA. \\ 2Department of Biomedical Engineering, University of North Carolina at Chapel Hill, Chapel Hill, \\ NC, 27599, USA.
}

\section{Abstract}

Murine embryonic limb cultures have invaluable roles in studying skeletogenesis. Substance delivery is an underdeveloped area in developmental biology that has primarily relied on Affi-GelBlue-Agarose-beads. However, the lack of information about the efficiency of agarose-bead loading and release and difficulties for a single-bead implantation represent significant limitations. We optimized the use of glycol-chitosan-5 $\beta$-cholanic-acid-conjugates (HGC) as a novel protein delivery system in mouse embryonic limbs. To this purpose, we loaded HGC either with recombinant Noggin, or bovine serum albumin (BSA). The size, morphology and stability of the protein-loaded-HGC were determined by transmission-electron-microscopy and dynamic-lightscattering. HGC-BSA and HGC-Noggin loading efficiencies were $80-90 \%$. Time-course study revealed that Noggin and BSA were $80-90 \%$ of released after 48 -hours. We developed several techniques to implant protein-loaded-HGC into murine embryonic joints from embryonic age (E)13.5 to E15.5, including a micro-injection system dispensing nanoliters. HGC did not interfere with skeletogenesis. Using CBR-3BA staining, we detected HGC-nanoparticles within implanted tissues. Furthermore, a sustained release of BSA and Noggin was demonstrated in HGC-BSA and HGC-Noggin injected regions. HGC-released Noggin was biologically active in blocking the BMP signaling in in vitro mesenchyme limb micromasses as well as in ex-vivo limb cultures. Results reveal that HGC is a valuable protein-delivery system in developmental biology.

\section{Keywords}

Embryonic mouse limb implant; Hydrophobically modified glycol chitosan; Protein delivery system; Developmental biology; Skeletogenesis

\footnotetext{
(C) 2009 Elsevier B.V. All rights reserved.

*Author of correspondence: University of North Carolina at Chapel Hill, Department of Pediatrics, Division of Pediatric Endocrinology, Mason Farm Road, Campus Box: 7039, Chapel Hill, NC, 27599-7039, USA. Phone: (919) 843-6905; spagnoa@med.unc.edu.

Publisher's Disclaimer: This is a PDF file of an unedited manuscript that has been accepted for publication. As a service to our customers we are providing this early version of the manuscript. The manuscript will undergo copyediting, typesetting, and review of the resulting proof before it is published in its final citable form. Please note that during the production process errors may be discovered which could affect the content, and all legal disclaimers that apply to the journal pertain.
} 


\section{Introduction}

Diffusible proteins play critical roles in developmental biology. EX-vivo cultures of dissected embryonic organs have been widely used and limb bud cultures in particular have played a critical role in unraveling pivotal steps of the skeletogenesis process.

Skeletogenesis within the developing limbs is a well organized process that arises from the condensation of cartilage progenitor cells into a cartilaginous template that is subsequently replaced by bone to form the adult skeletal elements separated by the joints. The synovial joints form through segmentation of the continuous cartilaginous template with loss of differentiated chondrocytes at the sites of the developing joints and emergence of a nonchondrocytic joint-forming cell population that undergoes condensation, flattens, and develops an interzone that then cavitates to form the joint space within the articular cartilage $[1,2]$. Joint interzone formation is known to begin at embryonic age (E) 13.5, but there is limited information on the mechanisms that regulate the complex multistep process. In fact, very few molecules have been reported to be necessary and/or sufficient to initiate the joint formation process (namely Noggin and growth and differentiation factor-5 [3, 4]). The mechanisms through which these molecules regulate the temporally and spatially organized emergence of joint interzone cells within the chondrogenic condensates are unclear.

One of the approaches to elucidate the mechanisms through which diffusible molecules regulate limb development is the local implantation of carriers containing the molecule by injection. Although,numerous micro-carrier materials have been used to deliver molecules into adult tissues, studies in embryonic tissues are limited and have primarily relied on AffiGel Blue beads [5, 6] or fibrous glass matrix [7, 8]. However, the lack of insight into protein loading efficiency and the dynamics of protein release pose some issues for the interpretation of the experimental data. Furthermore, the technical difficulties of implanting a single agarose bead (size in the order of 50-250 $\mu \mathrm{m}$ ) into mouse embryonic paws with sizes ranging from $1.0 \mathrm{~mm} \times 1.2 \mathrm{~mm}$ at E13.5 to $1.5 \mathrm{~mm} \times 1.8 \mathrm{~mm}$ at E16.5 represent critical limitations in the use of these materials. Nanoparticles may represent a novel approach for this area of developmental biology research that may overcome these limitations.

Kim et al. reported the preparation of hydrophobically modified glycol chitosan (HGC) nanoparticles consisting of glycol chitosan conjugated with $\beta$-cholanic acid [9-15]. HGC nanoparticles have been reported to encapsulate and sustainably release various chemicals and peptides [12-20]. Furthermore, the use of glycol chitosan derivatives is safe because these materials are biocompatible, biodegradable, and weakly immunogenic [21]. These characteristics, along with their size $(\sim 200 \mathrm{~nm})$ and adaptability for implants, make the HGC particularly suitable for delivering proteins into dissected embryonic limbs.

Here we describe: 1) HGC-protein loading, size, morphology and kinetics release of Noggin and bovine serum albumin (BSA); 2) the retained biological activity of Noggin after release from HGC; 3) the development of reliable micro-techniques for local implant of HGC as a carrier to study diffusible molecules into dissected murine limbs, including developing joints at different embryonic stages without perturbing development; 4) the detectability to HGC nanoparticles after histological sectioning; 5) the sustained release of diffusible proteins from implanted HGC into dissected embryonic limbs. Our data reveals the potential of HGC as a novel protein delivery system which can be used for research in developmental biology. 


\section{Materials and Methods}

\subsection{Materials}

Glycol chitosan $\left(\mathrm{MW}=2.5 \times 10^{5}\right.$; degree of deacetylation=82.7\%) was purchased from Sigma-Aldrich. It was dissolved in distilled water, filtered with a $22 \mu \mathrm{m}$ pore size filter to remove insoluble impurities. N-hydroxysuccinimide, $5 \beta$-cholanic acid, and 1-ethyl-3-(3dimethylaminopropyl)-carbodiimide hydrochloride were obtained from Sigma-Aldrich and used without further purification. Deionized distilled water (Millipore; resistanceN4M $\Omega$ ) was used for HGC synthesis and characterization. Recombinant mouse Noggin was purchased from R\&D and resuspended with BSA at a final concentration of $100 \mathrm{ng} / \mu \mathrm{l}$ for Noggin and $5 \mu \mathrm{g} / \mu \mathrm{l}$ for BSA to maintain the stability of the protein as recommended by the manufacturer. BSA was purchased from Sigma-Aldrich. All chemicals were analytical grade and used without further purification.

\subsection{Preparation of HGC}

HGC was prepared as previously reported [22]. Briefly, glycol chitosan $(2 \mu \mathrm{mol}, \mathrm{MW}=$ $\left.2.5 \times 10^{5}\right)$ was dissolved in distilled water $(60 \mathrm{~mL})$, followed by dilution with methanol (60 $\mathrm{mL})$ (water/methanol co-solvent $(1: 1 \mathrm{v} / \mathrm{v}) .5 \beta$-cholanic acid $(56 \mu \mathrm{mol}, \mathrm{MW}=360.57)$ was added and stirred until the solution was optically transparent. To activate carboxylic acid pertaining to $5 \beta$-cholanic acid, equal amounts ( 1.5 equiv $5 \beta$-cholanic acid]) of EDAC (84 $\mu \mathrm{mol}, \mathrm{MW}=191.70)$ and NHS $(84 \mu \mathrm{mol}, \mathrm{MW}=115.09)$ were added into the polymer solution, which allowed formation of the amide linkage by the reaction with primary amino groups in glycol chitosan. The resulting solution was stirred for 24 hours at room temperature, dialyzed (molecular weight cutoff $=10 \mathrm{kDa}$, Spectra Por) for 3 days against the excess amount of water/methanol mixture $(1 \mathrm{v} / 4 \mathrm{v})$, and lyophilized.

\subsection{Preparation and characterization of HGC-Noggin and HGC-BSA nanoparticles}

Proteins were encapsulated into HGC nanoparticles by a solvent evaporation method as previously reported with some modifications [14]. HGC (5 mg) was resuspended in $10 \mathrm{ml}$ solution containing distilled water:ethanol $=1: 1$, then mixed with $\mathrm{dH} 2 \mathrm{O}(0.17 \mathrm{ml})$ containing either BSA $(0.5 \mathrm{mg}$ ) or Noggin and BSA (respectively Noggin $10 \mu \mathrm{g}$ and BSA $0.5 \mathrm{mg}$ ), additional ethanol $(5 \mathrm{ml})$ was added to final ratio of distilled water:ethanol=1:2, to obtain HGC-BSA and HGC-Noggin. The solvents were then evaporated for 6-10 min at $60{ }^{\circ} \mathrm{C}$ using a rotary evaporator to prepare a thin polymer/peptide film. After addition of $5 \mathrm{ml}$ of distilled water, the solution was gently stirred for $10 \mathrm{~min}$ and then filtered $(0.8 \mu \mathrm{m}$ pore size filter). The resulting solution was purified using the Amicon Ultra-4 system (100 kDa cutoff membrane) (1600 rpm for about $30 \mathrm{~min}$ ), unfiltered and filtered solutions (containing unloaded "free" protein) were collected and lyophilized. Fractions were also collected at each of the preparation steps and a $25 \mu \mathrm{l}$ aliquot was used to determine either BSA or Noggin protein concentration using respectively the Bio-Rad DC protein assay reagents (Bio-Rad Laboratories) for the HGC-BSA preparation or Noggin western immunoblot (WIB) analysis for Noggin-HGC preparation. The morphology and size distribution of HGC nanoparticles, loaded or unloaded with proteins, were characterized using dynamic light scattering (DLS) (Zetasizer 32, Malvern Instruments) and transmission electron microscopy (TEM) (JEOL 100CX, located at the Microscopy Laboratory Services Core Facility at UNC). Five milligrams of HGC, HGC-BSA or HGC-Noggin polymer/peptide films were resuspended in $5 \mathrm{ml} \mathrm{dH} 2 \mathrm{O}$ that were either gently shaken for $6 \mathrm{~h}$ for HGC or gently stirred for 10 min for HGC-BSA and HGC-Noggin after evaporation. Thereafter, the solutions were sonicated 20 times ( 5 seconds on and 2 seconds off for each time) using a probe-type sonicator (Sonic Dismembrator Model 500, Fisher Scientific) at $90 \mathrm{~W}$ and filtered $(0.8 \mu \mathrm{m}$ pore size filter). DLS system was operated at $633 \mathrm{~nm}$ and $25 \pm 0.1^{\circ} \mathrm{C}$. The scattered light, generated by self-aggregates in distilled water was measured at an angle of $90^{\circ}$ and was 
colle cted with a BI-9000AT autocorrelator [23]. TEM images were taken at an accelerating voltage of $100 \mathrm{kV}$. Polymer solution $(5 \mu \mathrm{l})$ with a concentration of $1 \mathrm{mg} / \mathrm{ml}$ was placed onto a carbon-coated copper grid, taped with a filter paper to remove surface water, and air-dried for $5 \mathrm{~min}$. TEM measurements were carried out after the sample was negatively stained by $2 \%$ uranyl acetate [24].

\subsection{Noggin and BSA releases from HGC nanoparticles}

HGC-Noggin or HGC-BSA nanoparticles $(2 \mathrm{mg} / \mathrm{ml}$ ) were dispersed in PBS. The bulk solution $(600 \mu \mathrm{L})$ was placed into a cellulose membrane Float-A Lyzer (MW cutoff $=100$ $\mathrm{kDa}$ ). The Float-A Lyzer was placed in $30 \mathrm{ml}$ of PBS and gently shaken at $37^{\circ} \mathrm{C}$ in a water bath. At various time points, the medium was refreshed. Samples were taken at predetermined times and concentrated with Amicon Ultra-4 system $(5 \mathrm{kDa})$ into $600 \mu \mathrm{l}$. Samples were saved and $25 \mu \mathrm{l}$ solutions were used for protein concentration determination either by Bio-Rad DC protein assay for BSA or WIB analysis for Noggin.

\subsection{Proteins released from HGC to treat limb bud micromass cultures}

Limb bud micromass cultures were prepared as previously reported [25]. Briefly, limbs were collected aseptically from E13.5 embryos, teased apart into small pieces and incubated with dispase ( $1 \mathrm{U} / \mathrm{ml}$ in PBS) (GIBCO BRL) for $\sim 45$ minutes at $37^{\circ} \mathrm{C}$. Dispase-digestion was terminated by cell resuspension in culture medium (CM) (60\% Ham's F12, 40\% DMEM containing 10\% FBS). Cells were passed through a No. 20 Nitex (Tetko) mesh to remove cell clumps. Micromass cultures were initiated by carefully placing $10 \mu \mathrm{l}$ of the cell suspension $\left(1 \times 10^{5}\right.$ cells $)$ into the center of a 24 -well plate. Cultures were placed in a cell incubator at $37^{\circ} \mathrm{C}$ in $5 \% \mathrm{CO}_{2}$. After attachment $(\sim 1.5$ hour $), 1 \mathrm{ml}$ of $\mathrm{CM}$ was added and cultures were returned to the incubator. Media changes were performed at least once a day. To obtain HGC-released Noggin, $0.1 \mathrm{mg}$ of HGC-Noggin (containing $200 \mathrm{ng}$ ofNoggin), or HGC, or HGC-BSA were resuspended in $1 \mathrm{ml}$ serum free $\mathrm{CM}$ and placed in the incubator for two days. Collected media were filtered using a $100 \mathrm{kDa}$ Microcon (Millipore) and used to treat micromass cultures for 20 minutes.

\subsection{WIB analysis}

Fractions from Noggin loading assay and in vitro release assay as well as total protein micromass cell lysates were subjected to WIB using ECL reaction (Pierce) as previously reported [26]. Total protein cell lysates were obtained by solubilizing micromass cell cultures in 2X SDS sample buffer (20 mM dithiothreitol, 6\% SDS, $0.25 \mathrm{M}$ Tris, pH 6.8, $10 \%$ glycerol, $10 \mathrm{mM} \mathrm{NaF}$ and bromophenyl blue). Total protein concentration was measured using Bio-Rad DC protein assay; equal amounts of protein $(20 \mu \mathrm{g})$ were used for WIB analysis as previously reported [26]. Noggin and phospho-Smad-1,5,8 polyclonal antibodies were from Cell Signaling. Anti-beta-actin polyclonal antibody was from SigmaAldrich. Anti-rabbit and anti-mouse IgG, horseradish peroxidase (HRP)-conjugated secondary antibodies were from Amersham Bioscience.

\subsection{HGC-BSA nanoparticles implantation and ex-vivo limb cultures}

Limb autopods (paws) were dissected either from E13.5, E14.5 or E15.5 C57BL/6 mouse embryos in ice-cold PBS. Dissected limbs were half embedded inside 1.5\% agarose/PBS gel and placed under an Olympus SZX19 stereo-microscope. Two techniques were developed to perform HGC or HGC-BSA implants into discrete regions of the autopods. In the first technique, HGC or HGC-BSA suspensions were implanted using a graduated syringe mounted with a 33G needle delivering $\sim \mu 1$ to each limb. In the second technique, a microinjection unit (Picosprizer II) delivering $0.0015 \mu 1$ per injection was assembled. The micro-injection unit was mounted with glass micro-pipettes (Sutter Instrument) that were 
pulled using a P-30 puller (Sutter Instrument) (setting 700 for Heat\#1 and 200 for pull force). The tip of the pipette was cut to obtain a diameter of $50 \mu \mathrm{m}$. Injection was performed using high pressured nitrogen set at 36.5 PSI for 3 milliseconds. Using the micro-injector, small site implants (joint site) were performed by delivering 1-3 shots. For large site implants (metatars or metacarps, or whole paw), about 40 shots were delivered. Implanted limbs were cultured in BGJb medium (Gibco BRL) supplemented with penicillin/ streptomycin (Gibco BRL). The limbs were incubated in a tissue culture incubator. Images were taken right after injection or 2-3 days after injection/culture under a SZX19 stereomicroscope equipped with a DP71 digital camera (Olympus) and were imported into Photoshop (Adobe), where they were formatted without using any imaging enhancement. For timed pregnancies, noon of the day when evidence of a vaginal plug was found was considered E0.5. Protocols were approved by the University of North Carolina at Chapel Hill Institutional Animal Care and Use Committee.

\subsection{Cibacron Brilliant Red-3BA and Hematoxylin staining}

Cibacron Brilliant Red-3BA (Aldrich Chem.) $0.03 \%$ staining was performed according to the previously published protocol [27] with some modifications. Briefly, paraffin sections (6 $\mu \mathrm{m})$ and frozen sections $(20 \mu \mathrm{m})$ were obtained using standard procedures, as previously reported [2].Sections were hydrated with $\mathrm{ddH}_{2} \mathrm{O}$ and then stained with Hematoxylin for 3 minutes. After washing in running tap water for 5 minutes in a $2 \mathrm{~L}$ beaker and rinsed in 2 changes of $\mathrm{ddH}_{2} \mathrm{O}$ (2 minutes per change), sections were stained in CBR-3BA stain solution ( $\mathrm{pH}$ 3.2) for 3 minutes. After rinsing in 2 changes of $\mathrm{ddH}_{2} \mathrm{O}$ ( 2 minutes per change.), sections were dehydrated in 2 changes each of: $95 \%$ alcohol ( 2 minutes per change) and absolute alcohol ( 2 minutes per change). Finally, sections were washed in 3 changes ( 2 minutes per change) of CitriSolv and mounted with resinous medium.

\subsection{BSA, pSmad1,5,8 and Noggin immunohistochemistry (IHC)}

Anti-BSA-HRP antibody was from ABcam. Frozen sections were blocked with $2 \%$ normal goat serum in PBS-Tween for 1 hour at room temperature and incubated with anti-BSA$\mathrm{HRP}$ antibody at $4^{\circ} \mathrm{C}$ overnight. Sections were then incubated in avidin-biotin peroxidase complex solution from Vector Laboratories for 30 minutes at room temperature and peroxidase activity was visualized with a 3,3' diaminobenzidine substrate kit (Vector). AntipSmad1,5,8 antibody and anti-Noggin antibody were from Cell Signaling. Paraffin sections were used to perform pSmad1,5,8 and Noggin IHC according tomanufacturers' protocol.

\section{Results}

\subsection{BSA and Noggin can be loaded into HGC nanoparticles with high efficiency, size and morphology after loading}

It has been reported that nano-sized HGC can encapsulate and release small peptides (MW $\sim 600 \mathrm{Da})$ [17]. To determine whether HGC nanoparticles can encapsulate larger proteins, we used BSA (MW = 66,000 Da) and Noggin (MW = 33,000 Da). Self-assembled HGCNoggin embedded nanoparticles were prepared according to the strategy schematically presented in Figure 1A. To verify protein loading efficiency to HGC nanoparticles, fractions were collected (fractions designated as F1 to F4) at each of the preparation and purification steps. HGC-BSA nanoparticles were prepared similarly and fractions were subjected to quantitative colorimetric BSA assay. The loading efficiency of BSA to HGC, expressed as the percentage of the starting concentration $(\mathrm{F} 1)$ to the concentration detected in the final fraction ( $\mathrm{F} 4$ ), was determined to be $84 \pm 3 \%$ ( $\mathrm{n}=3$ distinct preparations). Fractions obtained during HGC-Noggin preparation were subjected to WIB. The single anti-Noggin reactive band detected was quantified by densitometric analysis (Figure 1B). Serial dilutions of native Noggin protein starting solution were used to generate an internal standard curve to 
semi-quantify concentration of Noggin in the fractions (Figure 1B). The loading efficiency of Noggin to HGC (percentage of F4/F1) was $93 \pm 4 \%$ ( $\mathrm{n}=3$ distinct preparations). Noggin protein was undetectable in the $\mathrm{F} 3$ fraction containing unloaded (free) protein, confirming the high loading efficiency of HGC for Noggin (Figure 1B). Results show that HGC nanoparticles are capable of high loading efficiency of the relatively high molecular weight proteins BSA and Noggin.

To determine the size and morphology of HGC after protein loading we performed DLS and TEM analyses. As shown in Figure 2, the mean diameter of HGC nanoparticles is $211 \pm 14$ $\mathrm{nm}(\mathrm{n}=3)$ (Figure 2A), HGC-BSA and HGC-Noggin (containing BSA) had larger mean diameters of $360 \pm 16 \mathrm{~nm}(\mathrm{n}=3)$ (Figure $2 B$ ) and $357 \pm 6 \mathrm{~nm}(\mathrm{n}=3)$, respectively (Figure $2 \mathrm{C}$ ). The similar diameters of HGC-BSA and HGC-Noggin nanoparticles is explained by the fact that both preparations were loaded with BSA with a ratio of Noggin:BSA of 1:50 for HGCNoggin. TEM analyses indicate that HGC-BSA or HGC-Noggin loaded nanoparticles display a spherical morphology similar to unloaded HGC but larger in size. To determine HGC-protein stability, DLS analyses were performed daily for 5 days on HGC, HGC-BSA and HGC-Noggin preparations at a physiological pH (in PBS, pH7.4) and maintained at 4 ${ }^{\circ} \mathrm{C}$. As shown in Figure S1, no significant changes in the average size were observed in the protein loaded and unloaded preparations.

\subsection{HGC nanoparticles demonstrate excellent properties for the sustained release of proteins in vitro}

To characterize the release of Noggin and BSA from HGC-Noggin and HGC-BSA respectively, we performed a time-course release profile by dialysis (100 kDa Floater-ALyzer). The protein release profile from HGC nanoparticles was determined in PBS (pH 7.4) at $37^{\circ} \mathrm{C}$, maintaining the protein gradient by regularly replacing the PBS dialysate solution at various time points (Figure 3A). Each solution was mixed with the previous and subjected to Noggin WIB analysis (Figure 3B). Noggin protein was quantified by densitometric analyses. Serial dilutions of recombinant Noggin solution were used to generate an internal standard curve to evaluate the concentration of Noggin (Figure 3C). The Noggin release profile showed that $87 \pm 5 \%$ ( $n=3$ distinct experiments) of the protein was released, from the HGC nanoparticles within 48 hours following linear kinetics; a minimal additional release was observed after that time ( $94 \pm 7 \%$ after 72 hours) (Figure 3C). BSA had a similar HGC release profile with $84 \pm 3 \%$ released after 48 hours. These results show that HGC nanoparticles comprise excellent properties for a sustained release of protein at a physiological $\mathrm{pH}$.

\subsection{Noggin retains biological activity after loading and release from HGC}

Noggin binding to BMPs depends on its ability to forms dimers [28]. Performing Noggin WIB under non-reducing conditions we showed that Noggin loaded into HGC remained capable of forming dimers (Figure 4A). Furthermore, in order to determine whether HGCreleased Noggin maintained its biological activity we performed a Smad-1,5,8 phosphorylation assay in limb bud mesenchyme micromasses. To obtain HGC-released Noggin, HGC-Noggin and control HGC were incubated in serum free media for 48 hours. Control media or Noggin-containing media $(200 \mathrm{ng} / \mathrm{ml})$ were collected, and used to treat limb bud micromasses for 20 minutes, after which cell lysates were collected and subjected to WIB for phosphorylated Smad-1,5,8. As a positive control, cells were treated under the same conditions with native Noggin $(200 \mathrm{ng} / \mathrm{ml})$. As shown in Figure 4B, we found that HGC-released Noggin inhibited Smad-1,5,8 phosphorylation similarly to native Noggin. These results suggest that the biological activities of Noggin are not impaired by encapsulation in the HGC carrier. 


\subsection{HGC-BSA nanoparticles can be implanted throughout the autopod mesenchyme without interfering with mice limb joint development}

To address if HGC nanoparticles can be used as a novel protein delivery system for ex-vivo research in developmental biology, we tested the properties of HGC-loaded protein injected into dissected limb buds. We first implanted HGC-BSA and HGC mixed with Methylene Blue (to mark the injected sites) into dissected E13.5 autopods (paws) or E14.5 autopods (Figure S2A) using a syringe mounted with a 33G needle. Injections were performed either in the carpal or the tarsal regions (Figure S2A). As shown in Figure S2B, after culturing the autopods for 2-3 days, Methylene Blue/HGC was detectable within the whole limb mesenchyme region (phalanges, carpal/tarsal and metacarpal/metatarsal regions) indicating that this injection technique can be used to deliver HGC-protein throughout the entire autopod. To determine whether the implanted HGC nanoparticles interfered with the limb skeletal development, we performed hematoxylin staining of E13.5 cultured limbs. As depicted in Figure S2B, we found that HGC as well as HGC-BSA did not interfere with the skeletal development of the digits, including joint development. However, in E13.5 we noted some damage at the site where the needle was inserted (Figure S2A, asterisk).

\subsection{HGC-BSA nanoparticles can be implanted in discrete regions and at different embryonic stages of developing autopods using a micro-injection system}

In order to achieve implantation of HGC-protein within discrete developing autopod regions (i.e. at the developing joint site) and to deliver a controlled volume (nanoliter per injection), we have assembled a micro-injection unit equipped with a micropipette puller (to prepare calibrated pipettes) and a PicoSpritzer fluid dispensing system, as described in the Methods section. To fit our implantation site needs, we pulled glass pipettes to a tip diameter of 50 $\mu \mathrm{m}$ after tip cutting, to approximately one seventh of the diameter of a $33 \mathrm{G}$ needle (Figure S3). Mounting the pulled pipettes into the fluid-dispensing system, we implanted HGC-BSA nanoparticles at either the proximal interphalangeal joint or the distal interphalangeal joints of E13.5 and E15.5 embryos (Figure 5). E13.5 is the embryonic stage when the joint starts to develop, forming an interzone within the phalange ossification centers; at E15.5 the joint space is fully developed. The proximal interphalangeal joint was identified as the site between the distal part of the metatarsal/metacarpal region and the ending of the interdigital space; the distal joint was identified as a distal bending within the developing phalange. Using the micro-injection system, we were able to implant HGC-BSA (Figure 5). The loaded pipette was gently inserted (insertion site marked as a red circle in Figure 5) into the digits along the distal or proximal direction with an angle of about $20-30^{\circ}$ for $\sim 0.1-0.2 \mathrm{~mm}$ and 1 to 3 shots $(0.0015-0.0045 \mu \mathrm{l})$ were injected (delivery site marked blue by Methylene Blue and indicated by black arrows in Figure 5). No fluid diffusion was noted in the digits adjacent to the injection site even after culturing. Using the micro-injection system, we were also able to inject a wide range of volumes (from $0.005 \mu \mathrm{l}$ up to $0.1 \mu \mathrm{l}$ ) of HGC-BSA into discrete regions of the developing limbs. Furthermore, HGC or HGC-BSA implantations did not cause any apparent tissue damage and did not interfere with the limb skeletal development (Figure 8) at any developmental stage tested. Our data demonstrate the versatility of our delivery system that allows varying volumes of HGC nanoparticles carrying proteins to be precisely implanted into discrete regions of developing limbs, at different embryonic limb development stages.

\subsection{Detection of HGC nanoparticles and BSA after implant of HGC-BSA in developing autopods by histological analysis}

To determine whether HGC and HGC-BSA nanoparticles implanted into developing limbs can be detected in histological sections we used CBR-3BA staining. E15.5 limbs were implanted with HGC, HGC-BSA or left non-injected and adjacent frozen sections were either subjected to CBR-3BA staining or BSA-immunohistochemistry (IHC) (Figure S4). 
We found that HGC and HGC-BSA nanoparticles implanted into developing autopods stained uniquely red with CBR-3BA, while no staining was detectable in limbs that were left non-injected (Figure 6A-C). To determine whether BSA was detectable within the developing limbs implanted with HGC-BSA we performed IHC for BSA. We reasoned that since BSA is not produced in rodents, BSA-IHC would detect only the BSA that is delivered by HGC. As shown in Figure 6D, we found that BSA was detected within the developing limb mesenchyme cells implanted with HGC-BSA (4 hours after implant), while no signal was detectable in limbs implanted only with HGC (Figure 6E) or left non-injected (Figure 6F). Smaller magnifications (20X) of the CBR-3BA staining and BSA-IHC are depicted in Figure S4. Data demonstrate that a relatively small volume of injected HGC can be detected using CBR-3BA staining. We also performed BSA-IHC analysis in E15.5 autopods that were cultured for 1 and 2 days after HGC-BSA implantation. As a control, autopods were injected with native BSA (lacking the HGC carrier) delivering the same injection volume and concentration. As shown in Figure 7 A-C, BSA released from HGC was detectable right after implantation (7A), and 1 day (7B) and 2 days (7C) after implant/culture. In autopods injected with native BSA (Figure 7 D-F), while BSA immunostaining was evident right after injection (7D) it was undetectable 1 day (7E) and 2 days (7F) after organ injection/culture. Our findings indicate that HGC is a suitable carrier for the sustained release of proteins in cultured autopods.

\subsection{Implanted HGC-Noggin is detectable and inhibits phosphorylation of Smad1,5,8 in ex- vivo cultured embryonic limbs}

To determine whether Noggin was released after implant into embryonic limbs and retained its biological activity, IHC for Noggin and Smad-1,5,8 was performed in adjacent paraffin sections obtained from E14.5 autopods injected either with HGC-Noggin (Figure 8B' and 8B") or HGC-BSA (Figure 8A' and 8A") and cultured for 12 hours after implantation. As shown in Figure 8, a strong positive immunostaining for Noggin was detected (Figure 8B', red arrows) in the HGC-Noggin-injected region of the limbs, while minimal staining (indicating the endogenous Noggin) was detected in the corresponding region injected with HGC-BSA (Figure 8A'). Phosphorylation of Smad1,5,8 was inhibited in the same region in which injected Noggin was detected (Figure 8B", black arrows), but not in the corresponding region injected with HGC-BSA (Figure 8A"). These findings indicate that Noggin was released in implanted/cultured limbs and was biologically active.

\section{Discussion}

In our studies we have: 1) shown that HGC nanoparticles allow high loading efficiency and sustained biologically unaltered release of the relatively high molecular weight proteins BSA and Noggin; 2) developed several techniques to inject HGC-proteins into dissected mouse embryonic limbs including assembling a micro-injector system; 3) demonstrated the versatility of our delivery techniques to dispense HGC-protein at different gestational ages and within discrete regions; 4) identified HGC nanoparticles in histological preparations of HGC-protein injected embryonic limb organs; 5) detected the release of BSA and Noggin from HGC in injected limb organs; 6) demonstrated that Noggin released from HGC retained its biological activity of blocking the BMP signaling in in vitro mesenchyme limb micromasses as well as in ex-vivo limb cultures.

Embryonic organ cultures have been crucial in unrevealing critical aspects of the development process. EX-vivo embryonic organ cultures are superior to in vivo transplantation or isolated cell cultures in many aspects. The cultured tissues can be manipulated in multiple ways, and their development can be continuously monitored. The culture conditions are reproducible, and the composition of the medium is known exactly and can be modified. Furthermore, the ex-vivo culture conditions allow analyses of the 
embryonic tissue interactions (i.e. epithelial-mesenchymal) and of the nature of the inductive signals. The potentials for embryonic organ cultures to study the local long-term effects of diffusible molecules in morphogenesis have been hampered by the use of limiting and uncharacterized protein carriers. The Affi-gel agarose beads have been widely applied [5, 29-32]. Beads are 50-250 $\mu \mathrm{m}$ in diameter, are routinely used for protein purification and are composed of crosslinked agarose mesh with covalently attached Cibachrome blue F3GA dye. Although these carriers have the ability to absorb peptides in solution, the kinetics of protein release after in vivo or ex-vivo implantation is basically unknown. Furthermore, considering the relatively large size of the beads, single bead implantation into mouse embryonic tissues at early developmental stage is problematic, and since the bead's size is highly variable, protein binding per bead is unpredictable. Similar issues can be raised for the studies that employed fibrous glass matrix containing proteins, although these carries can be more easily implanted [7, 8]. Here, we characterized the loading and release properties of HGC for BSA and Noggin and developed suitable techniques for local implantation into embryonic limbs. We used the embryonic limbs as a model because it allows to study the development of specific skeletal tissues, for example joints that are highly organized and develop through temporally and spatially well-defined morphogenic stages that are reproducible in the ex-vivo organ cultures.

Several studies have shown that HGC nanoparticles through enhanced permeability have efficient delivery properties for tissue targeting in vivo and in vitro [10,11, 13-15, 17-19, $22,33]$. Furthermore, the subcellular size of HGC, biocompatibility and biodegradability are additional valuable characteristics at a pharmacologically relevant level. Herein, we report a novel application of HGC in embryonic organ cultures and show that implantation of HGCproteins in a focal manner results in production of targeted, detectable and imageable levels of sustained released proteins that retain their biological activities.

Delivery of carriers into embryonic tissues for achieving prolonged availability and maintaining efficient local concentration needs to be performed using relatively small volumes (in the order of $\mu$ /nano-liters), in relatively small regions (in the order of $\mu \mathrm{m}$ ). We have assembled a micro-injection unit with those specific characteristics and set the delivery pressure and pipette caliber parameters that allow an efficient and reproducible implantation of HGC-nanoparticles into dissected embryonic limbs without interfering with development and without causing any damage. The bench unit can be easily assembled with commercially available equipment and requires limited space. In addition we have determined the appropriate suspension volume for HGC-protein that leads to an adequate viscosity for the injection with minimal diffusion at the site.

In summary, we have showen that $\mathrm{HGC}$ can be used as a valuable protein delivery system in developmental biology research.

\section{Supplementary Material}

Refer to Web version on PubMed Central for supplementary material.

\section{Acknowledgments}

This work was supported by an Arthritis Foundation Investigator Award to A. Spagnoli. We acknowledge Dr. Joseph De Simone Professor of Chemistry, University of North Carolina at Chapel Hill, for his assistance with the DLS studies. 


\section{References}

[1]. Archer CW, Dowthwaite GP, Francis-West P. Development of synovial joints. Birth Defects Res C Embryo Today. 2003; 69(2):144-155. [PubMed: 12955858]

[2]. Spagnoli A, O’Rear L, Chandler RL, Granero-Molto F, Mortlock DP, Gorska AE, Weis JA, Longobardi L, Chytil A, Shimer K, Moses HL. TGF-beta signaling is essential for joint morphogenesis. J Cell Biol. 2007; 177(6):1105-1117. [PubMed: 17576802]

[3]. Brunet LJ, McMahon JA, McMahon AP, Harland RM. Noggin, cartilage morphogenesis, and joint formation in the mammalian skeleton. Science. 1998; 280(5368):1455-1457. [PubMed: 9603738]

[4]. Storm EE, Huynh TV, Copeland NG, Jenkins NA, Kingsley DM, Lee SJ. Limb alterations in brachypodism mice due to mutations in a new member of the TGF beta-superfamily. Nature. 1994; 368(6472):639-643. [PubMed: 8145850]

[5]. Hayek A, Culler FL, Beattie GM, Lopez AD, Cuevas P, Baird A. An in vivo model for study of the angiogenic effects of basic fibroblast growth factor. Biochem Biophys Res Commun. 1987; 147(2):876-880. [PubMed: 2443140]

[6]. Ganan Y, Macias D, Duterque-Coquillaud M, Ros MA, Hurle JM. Role of TGF beta s and BMPs as signals controlling the position of the digits and the areas of interdigital cell death in the developing chick limb autopod. Development. 1996; 122(8):2349-2357. [PubMed: 8756280]

[7]. Nifuji A, Noda M. Local application of bone morphogenic protein on developing chick embryos using fibrous glass matrix as a carrier. Methods in molecular biology (Clifton, N.J. 2000; 135:465-469.

[8]. Kuboki Y, Saito T, Murata M, Takita H, Mizuno M, Inoue M, Nagai N, Poole AR. Two distinctive BMP-carriers induce zonal chondrogenesis and membranous ossification, respectively; geometrical factors of matrices for cell-differentiation. Connect Tissue Res. 1995; 32(1-4):219226. [PubMed: 7554920]

[9]. Park JH, Cho YW, Chung H, Kwon IC, Jeong SY. Synthesis and characterization of sugar-bearing chitosan derivatives: aqueous solubility and biodegradability. Biomacromolecules. 2003; 4(4): 1087-1091. [PubMed: 12857096]

[10]. Son YJ, Jang JS, Cho YW, Chung H, Park RW, Kwon IC, Kim IS, Park JY, Seo SB, Park CR, Jeong SY. Biodistribution and anti-tumor efficacy of doxorubicin loaded glycol-chitosan nanoaggregates by EPR effect. J Control Release. 2003; 91(1-2):135-145. [PubMed: 12932645]

[11]. Kim K, Kwon S, Park JH, Chung H, Jeong SY, Kwon IC, Kim IS. Physicochemical characterizations of self-assembled nanoparticles of glycol chitosan-deoxycholic acid conjugates. Biomacromolecules. 2005; 6(2):1154-1158. [PubMed: 15762689]

[12]. Yoo HS, Lee JE, Chung H, Kwon IC, Jeong SY. Self-assembled nanoparticles containing hydrophobically modified glycol chitosan for gene delivery. J Control Release. 2005; 103(1): 235-243. [PubMed: 15710514]

[13]. Kim JH, Kim YS, Kim S, Park JH, Kim K, Choi K, Chung H, Jeong SY, Park RW, Kim IS, Kwon IC. Hydrophobically modified glycol chitosan nanoparticles as carriers for paclitaxel. J Control Release. 2006; 111(1-2):228-234. [PubMed: 16458988]

[14]. Park JH, Kwon S, Nam JO, Park RW, Chung H, Seo SB, Kim IS, Kwon IC, Jeong SY. Selfassembled nanoparticles based on glycol chitosan bearing 5beta-cholanic acid for RGD peptide delivery. J Control Release. 2004; 95(3):579-588. [PubMed: 15023468]

[15]. Hwang HY, Kim IS, Kwon IC, Kim YH. Tumor targetability and antitumor effect of docetaxelloaded hydrophobically modified glycol chitosan nanoparticles. J Control Release. 2008; 128(1): 23-31. [PubMed: 18374444]

[16]. Jo HG, Min KH, Nam TH, Na SJ, Park JH, Jeong SY. Prolonged antidiabetic effect of zinccrystallized insulin loaded glycol chitosan nanoparticles in type 1 diabetic rats. Arch Pharm Res. 2008; 31(7):918-923. [PubMed: 18704336]

[17]. Kim JH, Kim YS, Park K, Kang E, Lee S, Nam HY, Kim K, Park JH, Chi DY, Park RW, Kim IS, Choi K, Chan Kwon I. Self-assembled glycol chitosan nanoparticles for the sustained and prolonged delivery of antiangiogenic small peptide drugs in cancer therapy. Biomaterials. 2008; 29(12):1920-1930. [PubMed: 18289669] 
[18]. Kim JH, Kim YS, Park K, Lee S, Nam HY, Min KH, Jo HG, Park JH, Choi K, Jeong SY, Park RW, Kim IS, Kim K, Kwon IC. Antitumor efficacy of cisplatin-loaded glycol chitosan nanoparticles in tumor-bearing mice. J Control Release. 2008; 127(1):41-49. [PubMed: 18234388]

[19]. Min KH, Park K, Kim YS, Bae SM, Lee S, Jo HG, Park RW, Kim IS, Jeong SY, Kim K, Kwon IC. Hydrophobically modified glycol chitosan nanoparticles-encapsulated camptothecin enhance the drug stability and tumor targeting in cancer therapy. J Control Release. 2008; 127(3):208218. [PubMed: 18336946]

[20]. Park K, Hong HY, Moon HJ, Lee BH, Kim IS, Kwon IC, Rhee K. A new atherosclerotic lesion probe based on hydrophobically modified chitosan nanoparticles functionalized by the atherosclerotic plaque targeted peptides. J Control Release. 2008; 128(3):217-223. [PubMed: 18457896]

[21]. Hirano S. Chitin and chitosan as novel biotechnological materials. Polym Int. 1999; 48:732-734.

[22]. Kwon JHPS, Chung H, Kwon IC, Jeong SY. Physicochemical Characteristics of Self-Assembled Nanoparticles Based on Glycol Chitosan Bearing 5-beta-Cholanic Acid. Langmuir. 2003; 19:10188-10193.

[23]. Hyung Park J, Kwon S, Lee M, Chung H, Kim JH, Kim YS, Park RW, Kim IS, Bong Seo S, Kwon IC, Young Jeong S. Self-assembled nanoparticles based on glycol chitosan bearing hydrophobic moieties as carriers for doxorubicin: in vivo biodistribution and anti-tumor activity. Biomaterials. 2006; 27(1):119-126. [PubMed: 16023198]

[24]. Gratton SE, Napier ME, Ropp PA, Tian S, DeSimone JM. Microfabricated particles for engineered drug therapies: elucidation into the mechanisms of cellular internalization of PRINT particles. Pharm Res. 2008; 25(12):2845-2852. [PubMed: 18592353]

[25]. Cash DE, Bock CB, Schughart K, Linney E, Underhill TM. Retinoic acid receptor alpha function in vertebrate limb skeletogenesis: a modulator of chondrogenesis. J Cell Biol. 1997; 136(2):445457. [PubMed: 9015314]

[26]. Longobardi L, O’Rear L, Aakula S, Johnstone B, Shimer K, Chytil A, Horton WA, Moses HL, Spagnoli A. Effect of IGF-I in the chondrogenesis of bone marrow mesenchymal stem cells in the presence or absence of TGF-beta signaling. J Bone Miner Res. 2006; 21(4):626-636. [PubMed: 16598383]

[27]. Rossomacha CDHE, Shive MS. Simple Methods for Staining Chitosan in Biotechnological Applications. J Histotech. 2004; 27(1):31-36.

[28]. Groppe J, Greenwald J, Wiater E, Rodriguez-Leon J, Economides AN, Kwiatkowski W, Affolter M, Vale WW, Belmonte JC, Choe S. Structural basis of BMP signalling inhibition by the cystine knot protein Noggin. Nature. 2002; 420(6916):636-642. [PubMed: 12478285]

[29]. Schreiber AB, Winkler ME, Derynck R. Transforming growth factor-alpha: a more potent angiogenic mediator than epidermal growth factor. Science. 1986; 232(4755):1250-1253. [PubMed: 2422759]

[30]. Storm EE, Kingsley DM. GDF5 coordinates bone and joint formation during digit development. Dev Biol. 1999; 209(1):11-27. [PubMed: 10208739]

[31]. Thakur A, Lansford R, Thakur V, Narone JN, Atkinson JB, Buchmiller-Crair T, Fraser SE. Gene transfer to the embryo: strategies for the delivery and expression of proteins at 48 to 56 hours postfertilization. Journal of pediatric surgery. 2001; 36(8):1304-1307. [PubMed: 11479882]

[32]. Hayamizu TF, Sessions SK, Wanek N, Bryant SV. Effects of localized application of transforming growth factor beta 1 on developing chick limbs. Dev Biol. 1991; 145(1):164-173. [PubMed: 2019321]

[33]. Nam HY, Kwon SM, Chung H, Lee SY, Kwon SH, Jeon H, Kim Y, Park JH, Kim J, Her S, Oh YK, Kwon IC, Kim K, Jeong SY. Cellular uptake mechanism and intracellular fate of hydrophobically modified glycol chitosan nanoparticles. J Control Release. 2009; 135(3):259_ 267. [PubMed: 19331853] 


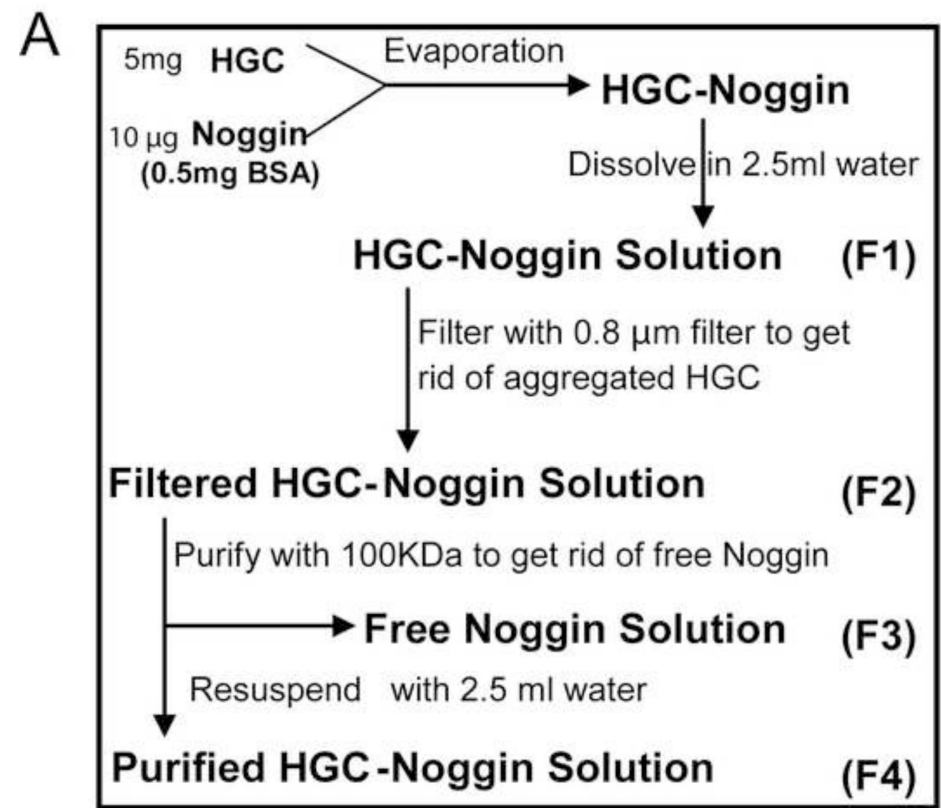

B

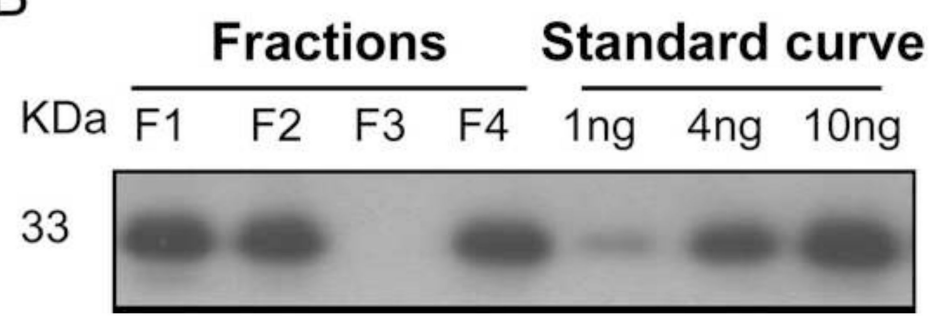

Figure 1.

HGC-Noggin preparation. (A) Schematic representation of the strategy used to obtain purified HGC-Noggin preparation. (B) Noggin WIB of fractions obtained during the loading-purification process. Starting solution (F1), after loading-evaporation (F2), unloaded "free" fraction (F3) and loaded fraction (F4). To evaluate the concentration of Noggin in the fractions, an internal standard curve using serial dilutions of recombinant Noggin was used. 

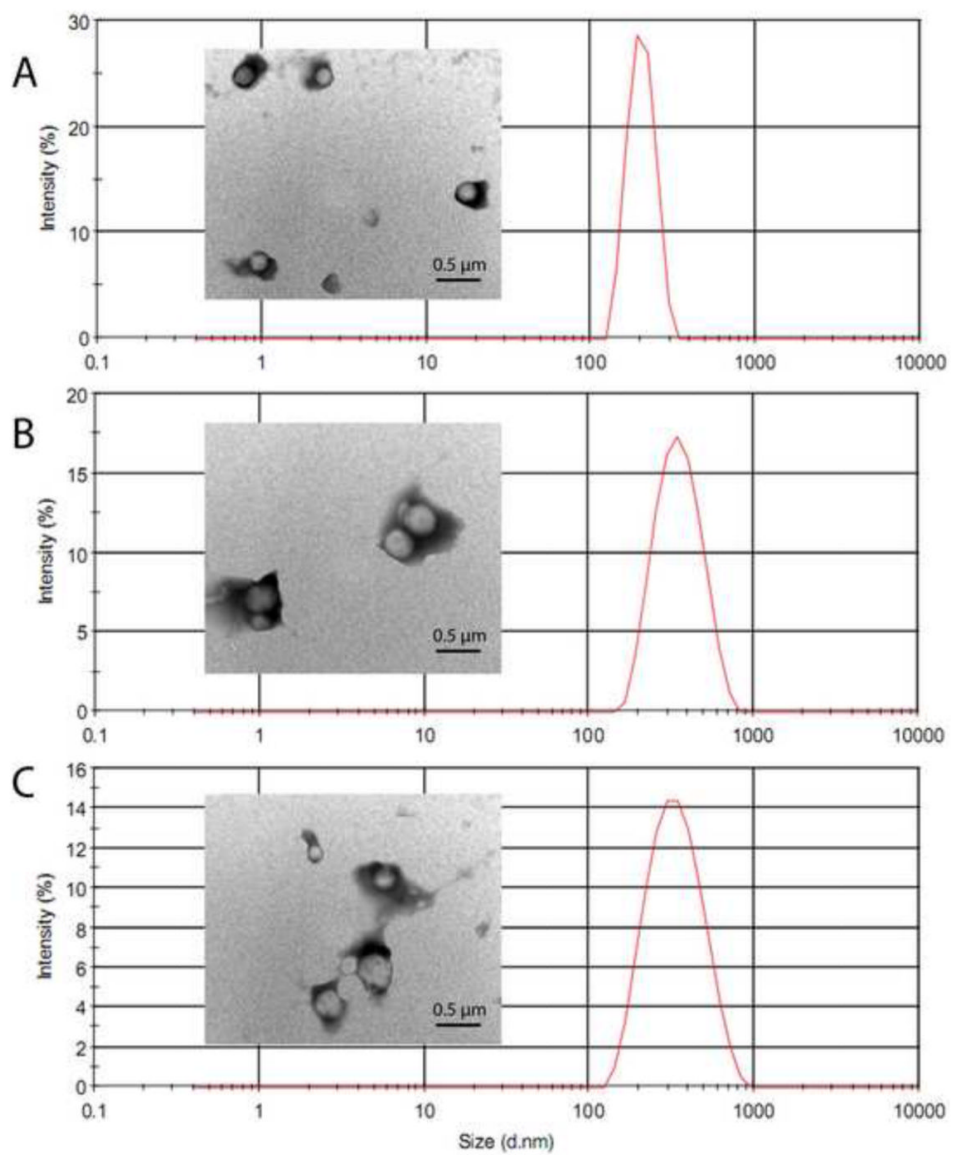

Figure 2.

Size distribution and morphology of (A) HGC nanoparticles, (B) HGC-BSA nanoparticles and (C) HGC-Noggin(BSA) nanoparticles, determined by DLS and TEM analyses. 


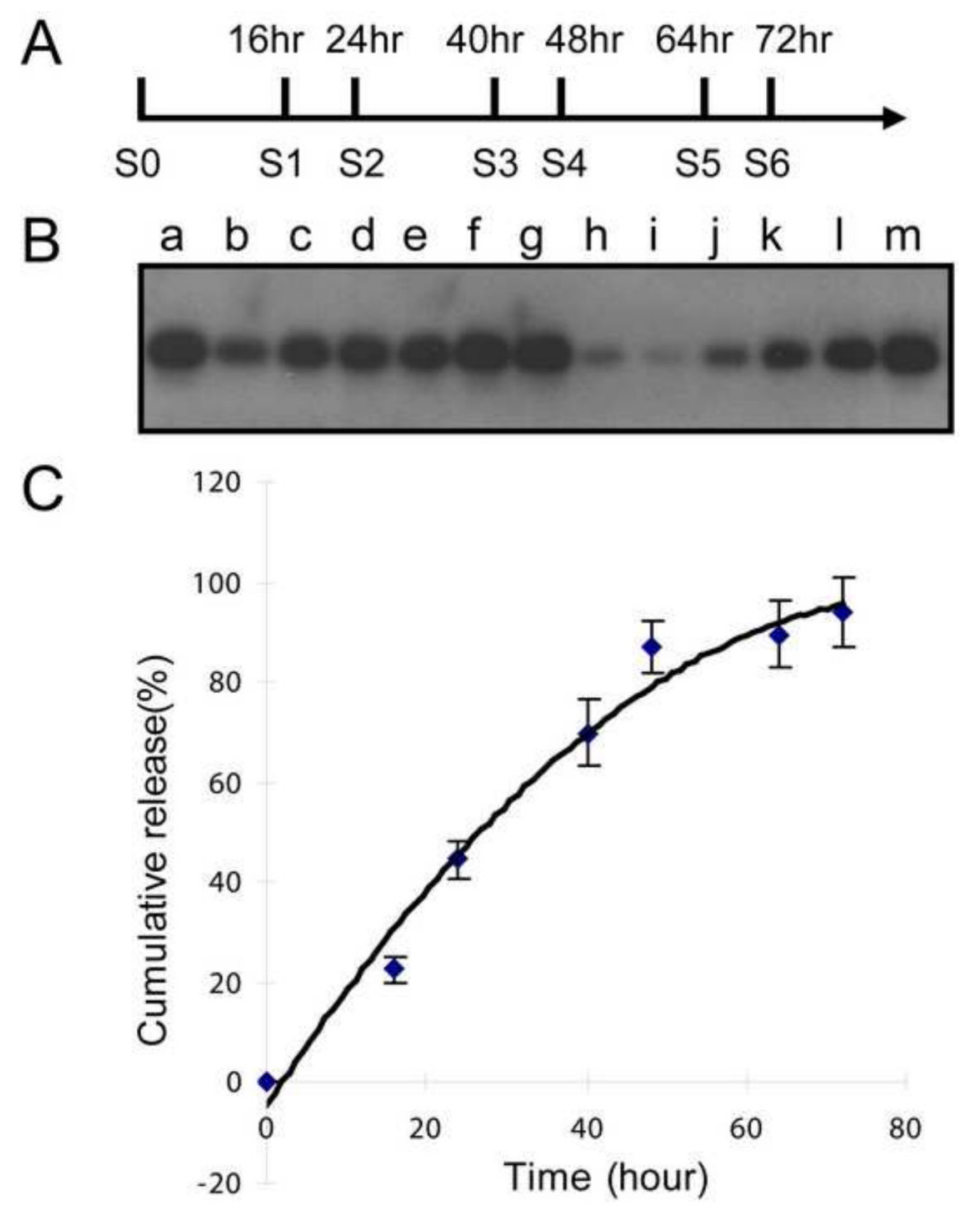

Figure 3.

Time-course analysis of Noggin release from HGC. (A) Refreshing dialyzing solution was obtained at the indicated time points. (B) Time-course of HGC-Noggin release by Noggin WIB analysis. a, HGC-Noggin, before release (expected 20ng); b, Released Noggin after 16 hours, S1; c, Released Noggin after 24 hours, S1+S2; d, Released Noggin after 40 hours, $\mathrm{S} 1+\mathrm{S} 2+\mathrm{S} 3$; e, Released Noggin after 48 hours, S1+S2+S3+S4; f, Released Noggin after 64 hours, S1+S2+S3+S4+S5; g, Released Noggin after 72 hours, S1+S2+S3+S4+S5+S6; h, Remaining Noggin; i-m, Noggin Standard curve: 1ng, 2ng, 5ng, 10ng, 20ng. (C) Release profile of Noggin from HGC-Noggin nanoparticles; three distinct data sets were obtained from respective WIB analyses that were averaged and represented as mean \pm standard deviation. 


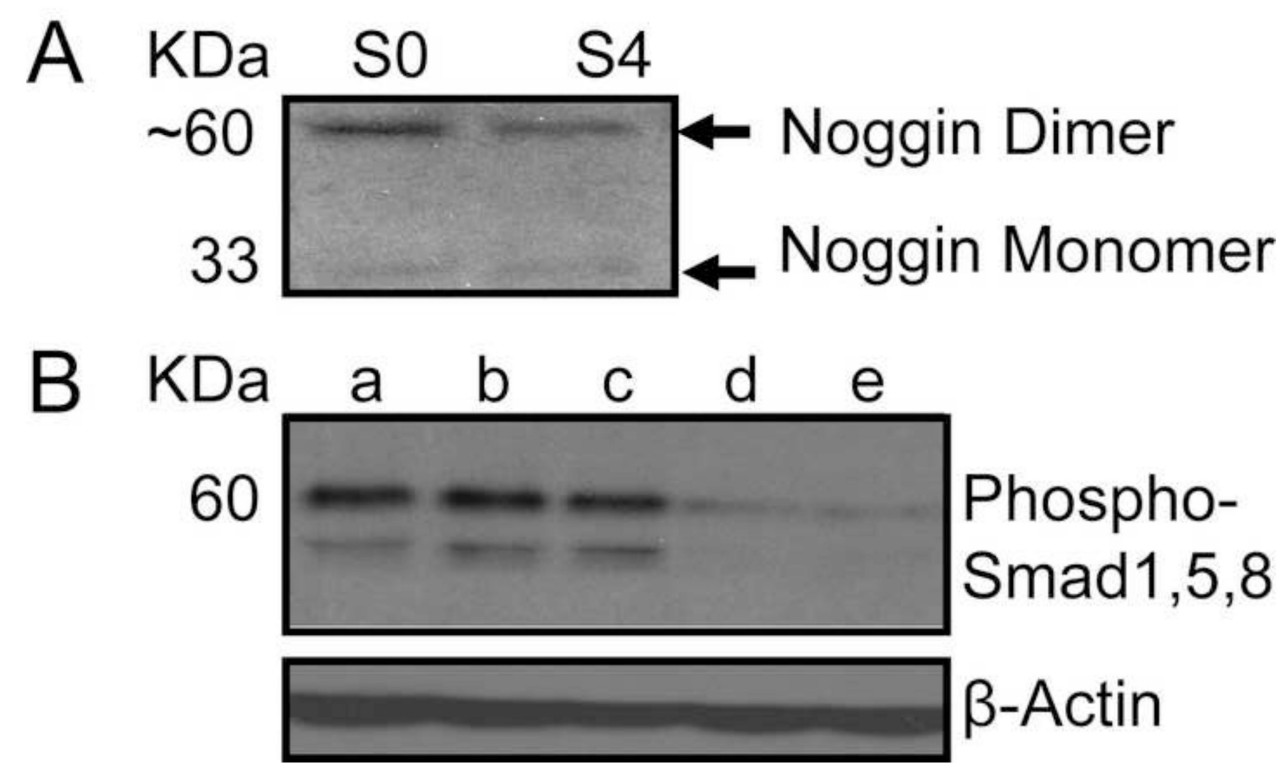

Figure 4.

Noggin loaded and released from HGC maintains its biological activity. (A) HGC-Noggin maintains the ability to form dimers as determined by Noggin WIB under non-reducing conditions. (S0): native Noggin; (S4): Noggin loaded to HGC. (B) Noggin released from HGC inhibits Smad-1,5,8 phosphorylation. HGC-Noggin, HGC-BSA, HGC alone were incubated for 48 hours in SF medium that was collected, dialyzed and used to treat limb mesenchyme micromasses for 20 minutes. a, control medium; $b$, medium from HGC alone; c, medium from HGC-BSA; d, medium from HGC-Noggin; e, $200 \mathrm{ng}$ of native Noggin 


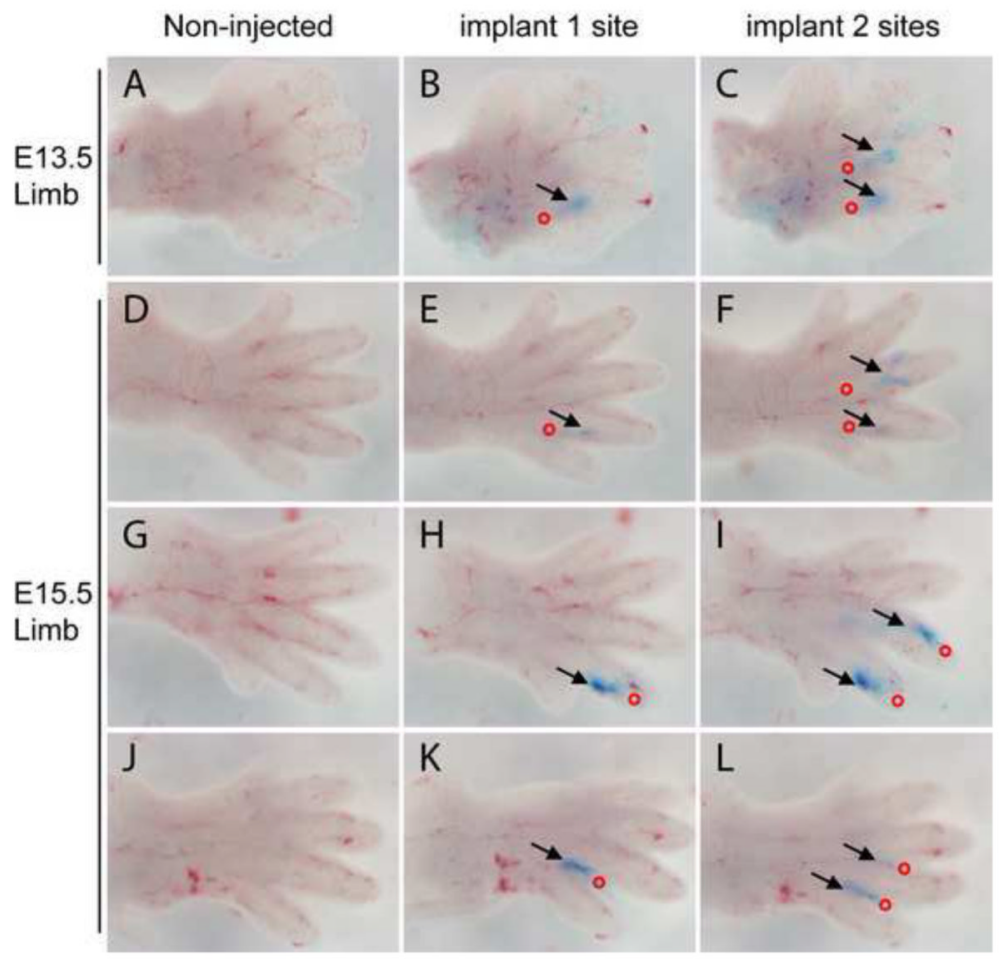

\section{Figure 5.}

HGC-BSA nanoparticles can be implanted into developing autopods using a microinjection unit. Dissected autopods from wild type C57BL/6 mice at either E13.5 (A-C) or E15.5 (D-L) were imaged before injection (non-injected, left panels) and then subjected to Methylene Blue-HGC-BSA implantation at one site (middle panels) followed by a second site (right panels). Red circles indicate the position where the pipette was inserted. Black arrows indicate where the Methylene Blue-HGC-BSA implants were delivered. Picture was taken immediately after delivery. See text for more details. 
HGC-BSA
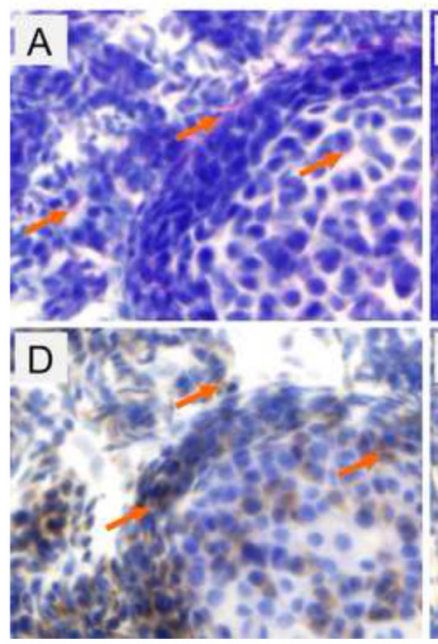

HGC
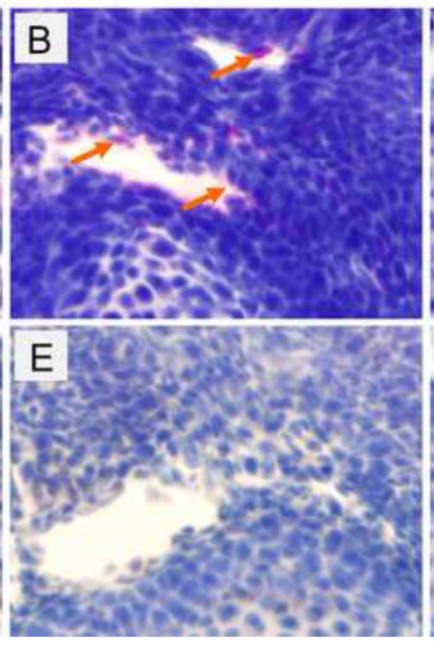

Non-injected
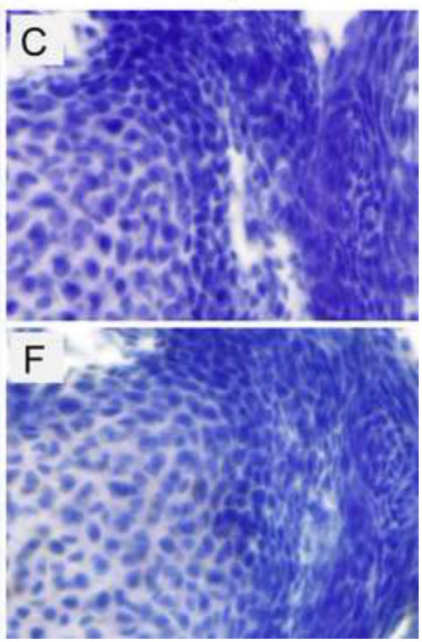

Figure 6.

Implanted HGC-BSA can be visualized with CBR-3BA staining and IHC for BSA. CBR-3BA staining (A-C) was performed on frozen sections from wild type C57BL/6 mice at E15.5 implanted either with HGC-BSA (A) or HGC (B) or left non-injected (C). IHC for BSA was performed on adjacent frozen sections (D-F). 100X magnifications are depicted. Pictures of unsectioned limbs as well as 20X magnifications including the region from where the 100X magnifications were obtained can be found in Figure S4 


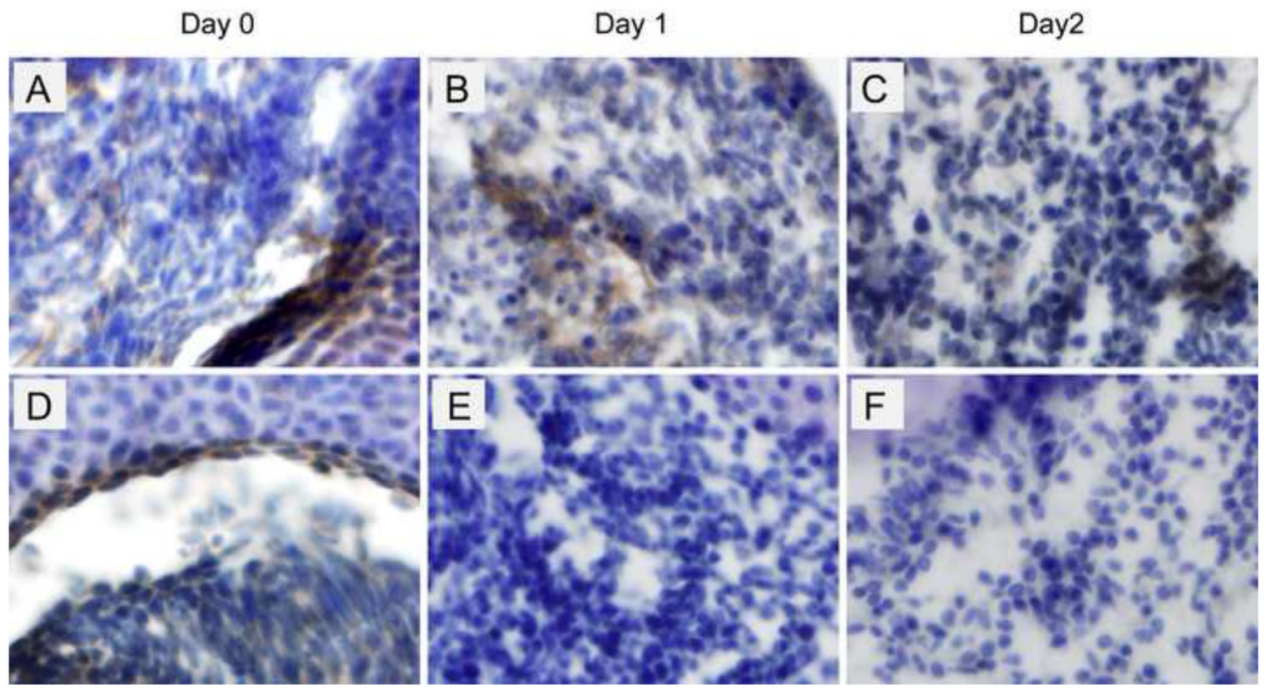

Figure 7.

Implanted HGC-BSA showed sustained BSA release. BSA IHC was performed on frozen sections from wild type C57BL/6 mice at E15.5 implanted either with HGC-BSA (A-C), or native BSA (D-F) and cultured for 1 and 2 days. 100X magnifications are depicted. 

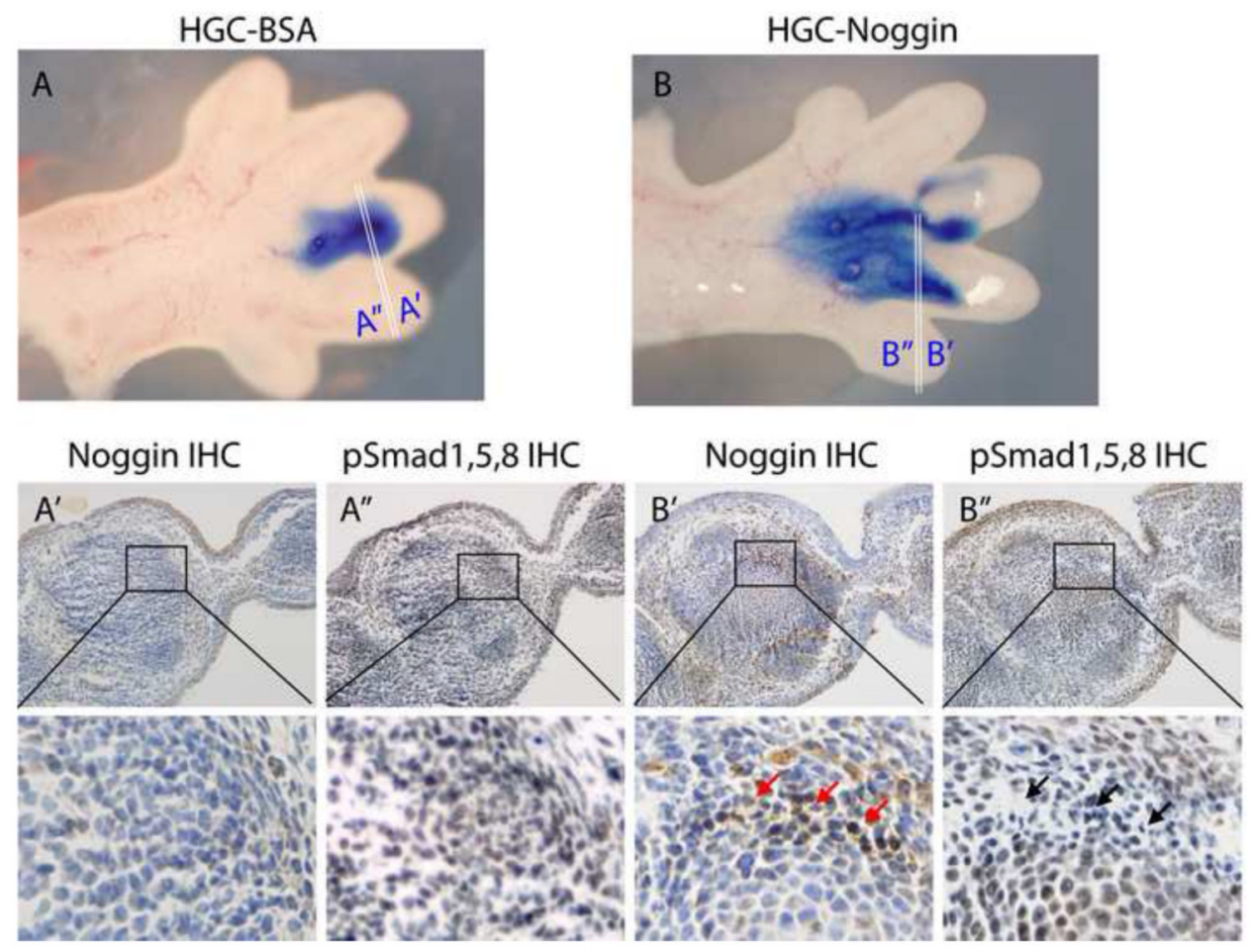

Figure 8.

HGC-Noggin is released and inhibits Smad-1,5,8 phophorylation after implantation into embryonic limbs and ex-vivo culturing. Noggin IHC and pSmad1,5,8 IHC were performed on adjacent paraffin sections obtained from E14.5 autopods implanted either with HGCBSA (A) or HGC-Noggin (B). 20X and 100X magnifications are depicted. Red arrows indicate Noggin IHC and black arrows indicate inhibited Smad-1,5,8 phosphorylation in the same region. 